\title{
Corpus
}

Alice KRIEG-PLANQUE. - La notion de " formule » en analyse du discours. Cadre théorique et méthodologique. Besançon : Presses Universitaires de FrancheComté, 2009, 145 pages.

\section{Damon Mayaffre}

\section{(2) OpenEdition}

Journals

Édition électronique

URL : http://journals.openedition.org/corpus/1775

DOI : $10.4000 /$ corpus. 1775

ISSN : 1765-3126

Éditeur

Bases; corpus et langage - UMR 6039

Édition imprimée

Date de publication : 15 novembre 2009

ISSN : 1638-9808

Référence électronique

Damon Mayaffre, "Alice kRieg-PLanaue. - La notion de «formule » en analyse du discours. Cadre théorique et méthodologique. Besançon : Presses Universitaires de Franche-Comté, 2009, 145 pages. », Corpus [En ligne], 8 | 2009, mis en ligne le 01 juillet 2010, consulté le 10 décembre 2020. URL : http:// journals.openedition.org/corpus/1775; DOI : https://doi.org/10.4000/corpus.1775

Ce document a été généré automatiquement le 10 décembre 2020.

(c) Tous droits réservés 


\title{
Alice KRIEG-PLANQUE. - La notion de " formule " en analyse du discours. Cadre théorique et méthodologique. Besançon : Presses Universitaires de Franche-Comté, 2009, 145 pages.
}

\author{
Damon Mayaffre
}

1 Dans un format éditorial accessible à tous (127 pages + index et bibliographie) et pour une somme modique (9 euros), Alice Krieg-Planque propose un ouvrage dont la lecture apparaît utile à tous ceux qui étudient les discours et leur circulation dans l'espace public, réfléchissent sur le rapport entre langage et société, s'interrogent sur le cadre interdisciplinaire de l'analyse du discours aujourd'hui.

2 S'appuyant sur sa thèse remarquée Emergence et emplois de la formule "purification ethnique » dans la presse française (1980-1994). Une analyse de discours, soutenue en 2000 et parue aux éditions du CNRS en 2003, l'auteure définit la notion de formule et la fonde comme une notion clef de l'AD. Ce faisant, A. Krieg-Planque en profite, dans de précieux chapitres liminaires, pour revisiter le cadre conceptuel de l'AD (chapitre 1) et payer sa dette envers les grands auteurs tels J. Dubois, M. Tournier, J. Guilhaumou, etc., et plus longuement, en ce qui concerne l'émergence de la notion de formule comme concept scientifique, à J.-P. Faye (chapitre 2) et à M. Ebel et P. Fiala (chapitre 3).

3 La formule est d'abord définie, dès l'introduction, en termes généraux comme « un ensemble de formulations qui, du fait de leurs emplois à un moment donné et dans un espace public donné, cristallisent des enjeux politiques et sociaux que ces expressions contribuent dans le même temps à construire » (p. 7). Mais c'est le chapitre 4, central dans l'ouvrage, qui caractérise précisément la notion par quatre dimensions.

4 - Son figement: au-delà (ou en deçà) des débats linguistiques, ouverts par Saussure même, sur la définition du figement, une formule se caractérise toujours par une stabilité du signifiant. Cette stabilité est la condition matérielle de reprise et de 
circulation de la formule. De fait, le figement apparait comme une évidence dans le cadre d'unités lexicales simples - car une formule est parfois composée d'un mot unique («mondialisation», «crise», «reprise», etc.). Il peut apparaître plus problématique en revanche dans le cadre d'unités complexes ("extrême droite", «blanc bonnet et bonnet blanc », etc.).

5 - Sa dimension discursive : moins que "linguistique ", la formule est une "notion discursive » (p. 84), c'est-à-dire que c'est l'usage social qui la construit et l'institue en formule, et non quelques codes ou règles linguistiques prévisibles. En cela, une formule se traite en discours, c'est-à-dire en pensant la tension réflexive entre le texte et le contexte socio-historique, le discours et les faits politiques. En ce sens aussi, si l'on excepte le cas de néologismes, la forme matérielle de ce qui pourra devenir une formule préexiste en langue et en discours ("prolifération nucléaire ", " mouvement social ", etc.) mais ne devient formule qu'à la faveur d'une pratique langagière donnée dans une situation sociolinguistique ou, au sens large, historique, bien identifiée.

6 - Son statut de référent social : par là, l'auteure insiste après [Fiala et Ebel 1983] sur une forme d'évidence voire d'impérialisme sociolinguistique de la formule, qui à un moment précis contraint le débat public, fait référence ou consensus, devient incontournable pour chacun et reconnaissable par tous. Ainsi ne pourra-t-on pas éviter les formules « développement durable » ou «énergie renouvelable » aujourd'hui dans le débat sur l'écologie. Quand bien même un locuteur refuserait ces formules, il se situerait encore par rapport à elle ; quand bien même il les dénoncerait, il participerait à leur circulation.

7 - Son aspect polémique : il s'agit là de la caractéristique le plus difficile à percevoir et pourtant la plus importante. Derrière une stabilité du signifiant - et A. Krieg-Planque insiste sur la caractérisation matérielle de la formule que le linguiste pourra repérer sur la chaîne -, la formule cache - et a peut-être pour fonction de cacher - une instabilité et une polimicité du signifié. Derrière la formule se dissimulent en effet toujours des enjeux et souvent des questions essentielles, parfois tragiques, irrésolues : "épuration ethnique ", "mondialisation ", "salaire de misère ", " commerce équitable ». Par l'euphémisme parfois, une nominalisation ou une adverbialisation, la société semble s'accorder sur un signifiant stabilisé pour désigner des rapports de domination intolérables ou nommer l'innommable.

8 Sur les bases de ces quatre caractéristiques, Alice Krieg-Planque termine enfin son ouvrage par un chapitre conclusif sur les enjeux de la notion de formule dans le panorama scientifique actuel. Nous retiendrons de ce chapitre 5 , le développement sur la construction de l'espace public et le rôle des médias dans cette construction; plus directement, sur le rôle des médias dans la mise en circulation des formules qui ellesmêmes participent $\grave{a}$ et de la construction de l'espace public. L'auteure fait alors jouer sa double compétence de linguiste et de scientifique de l'information et de la communication. Dans un propos nuancé, elle montre ainsi qu'une des conditions d'existence des formules est leur circulation tous azimuts et leur acceptabilité par tous. Par là, le rôle des médias en tant que caisse de résonance et principal artisan de la doxa, dans nos sociétés contemporaines, ne peut-être nié. Pourtant, il ne saurait suffire pour expliquer le phénomène. Et l'auteure de s'amuser à montrer que les formules sont rarement à l'initiative des journalistes eux-mêmes mais plus souvent le fait des hommes politiques ou des acteurs sociaux. 
9 Si l'ouvrage est utile, c'est qu'en peu de pages, il offre clef en main un outil théorique et pratique pour les analyses de discours. Notons du reste que si l'entrée «formule » ne figure pas dans le dictionnaire de [Détrie, Siblot, Vérine (dir.) 2001], elle existe déjà dans le dictionnaire de [Charaudeau et Maingueneau (dir.) 2002]. Surtout, l'ouvrage apparaît en 2009 comme un digne héritier et une continuation des publications de l'Ecole française du discours. Après un glorieux passé, celle-ci semble grâce à des chercheuses comme Alice Krieg-Planque connaître un nouveau souffle.

\section{BIBLIOGRAPHIE}

Charaudeau P., Maingueneau D. (dir.) (2002). Dictionnaire d'analyse du discours. Paris : Seuil. Entrée « formule » par Pierre Fiala.

Détrie C., Siblot P., Vérine B. (dir) (2001). Termes et concepts pour l'analyse du discours. Une approche praxématique. Paris : Champion.

Faye J.-P. (1972). Langages totalitaires. Paris : Hermann.

Fiala P., Ebel M. (1983). Langages xénophobes et consensus national en Suisse (1960-1981) : discours institutionnels et langage quotidien; la médiatisation des conflits. Université de Neuchatel, Faculté des lettres, 432 pages.

Krieg-Planque A. (2003). « Purification ethnique ». Une formule et son histoire. Paris : CNRS éditions.

\section{AUTEUR}

DAMON MAYAFFRE

CNRS, Université Nice-Sophia Antipolis 